\title{
Four-Channel Teleoperation with Time-Varying Delays and Disturbance Observers
}

\author{
Emma Delgado, ${ }^{1}$ Pablo Falcón, ${ }^{1}$ Miguel Díaz-Cacho, ${ }^{2}$ and Antonio Barreiro ${ }^{1}$ \\ ${ }^{1}$ Higher Technical School of Industrial Engineering, University of Vigo, 36310 Vigo, Spain \\ ${ }^{2}$ Higher Technical School of Computer Engineering, University of Vigo, 32004 Orense, Spain \\ Correspondence should be addressed to Emma Delgado; emmad@uvigo.es
}

Received 3 June 2015; Revised 17 July 2015; Accepted 26 July 2015

Academic Editor: Yuqiang Wu

Copyright (C) 2015 Emma Delgado et al. This is an open access article distributed under the Creative Commons Attribution License, which permits unrestricted use, distribution, and reproduction in any medium, provided the original work is properly cited.

\begin{abstract}
This paper addresses the robust stability of teleoperated systems under the four-channel architecture, affected by time-varying communication delays and using disturbance observers. It is based on our previous work which provides a framework for robust stability against delays with bounded variation and a bounded time-derivative, using structured singular values (SSV). The main new feature here is the inclusion of disturbance observers (DOBs). The DOB concept is well-documented and relevant to many applications, since only position (but not force) measurements are usually available. In this paper, we adapt two DOBs (master and slave) to our generic framework, by representing them as stable, fast filters affected by the uncertainty in the plant modelling. Our main result is an SSV test to verify robust stability. The simulation results confirm the usefulness of this approach.
\end{abstract}

\section{Introduction}

A teleoperation system consists of master and slave mechanical systems, where the master is directly manipulated by a human operator and the slave, operating in a remote environment, is designed to track the master closely. The main aspects when analyzing and designing these systems are stability and transparency, where transparency is the measure of how much the operator's actions are mirrored in the remote environment in an ideal situation. In practice, there is a compromise between these two goals mainly due to the presence of time delays generated by the communication channel [1].

As far as stability is concerned, the teleoperation schemes are classified [2] as intrinsically stable schemes (passivitybased) and delay-dependent stable schemes. In early works dealing with constant delay, this issue was addressed by means of frequency Laplace or passivity techniques, applied to linear time invariant master-slave two-port systems [15]. On the other hand, the most successful control scheme in achieving a fully transparency under ideal conditions for transparency properties is the four-channel control scheme $[1,2,4,6]$.
Moreover, the low cost and wide availability of the Internet has opened a new line of research to establish Internet-based teleoperation [6-8] which requires the control signal to be transmitted through the network, exposing the system control loop to the varying time delay of a packet switched network [9]. Some examples of actual bounds for the delay magnitude and its derivative, appearing with UDP (user datagram protocol) for different Internet locations can be seen in [8].

Furthermore, in [10] the authors affirm that the bilateral teleoperators designed within the passivity framework, using concepts of scattering and two-port network theory, provide robust stability against constant delay in the network and velocity tracking but cannot guarantee position tracking in general. That is why many recent results try to extend passivity-based architecture to solve these problems; see the tutorial $[11,12]$.

Another option is to use delay-dependent stability tools. With these, better performance, such as zero tracking error, is gained in the case of small network delays (the typical operating condition), although the possibility of losing stability when there are large delays must be accepted. Thus 
the natural solution is to use tools that check this fact and provide estimations of the maximum allowable delays.

In this sense, several efforts are being made in the development of delay-dependent stability tools like those in $[6,13]$ and references therein. We develop in [8] a generic approach to model any teleoperation setup as a negative single feedback loop containing a linear time invariant block and an uncertain time-varying delay. The main added value of this approach is the possibility of deriving frequencydomain conditions for robust stability in the presence of time-varying delays and parametric uncertainties. As a case study, the two-channel position error (PE) control scheme was tested for the Internet-based haptic teleoperation of a laboratory 3D-Crane. In [14], using this approach, we present preliminary results about the scaled four-channel control scheme for teleoperating manipulators. The result of applying this analysis technique is that we can justify the design of a $\gamma$-4C scheme previously used in [6], incorporating a tuning factor $\gamma$ to increase in practical conditions the stable region fixing the desired bounds on time-varying delay and parametric uncertainty, with the particularity of maintaining the tracking properties provided by this transparent control scheme.

In this work, because we know that force observers can be included using the concept of disturbance observer (DOB) [15-17], also called reaction force observers (RFOB) [18], to cope with unmeasured human or environmental forces, we adapt two DOBs (master and slave) to our generic framework for robust stability, by representing them as stable, fast filters affected by the uncertainty in the plant modelling. Our main result is an SSV test to verify robust stability for four-channel teleoperation with time-varying delays and disturbance observers.

The paper is organized as follows. Section 2 introduces an overall description of teleoperation system and Section 3 explains a generic model for robust stability analysis in delaydependent control schemes with disturbance observers. Section 4 defines a robust stability condition based on the generic model formulation. Section 5 describes the teleoperation case study. Section 6 then presents the analysis and simulation results. Finally, the conclusions are discussed in Section 7.

\section{Teleoperation System Description}

Consider a generic teleoperation setup, like that in Figure 1, consisting of the master side (left) and the slave side (right) exchanging signals through a communication channel.

The master and slave will be modeled as a mechanical system with coordinates (positions) given by the $n \times 1$ vectors $x_{m}, x_{s}$. In the Laplace domain, if $x_{m}(0)=\dot{x}_{m}(0)=0$, we have

$$
\begin{gathered}
P_{m}(s) X_{m}(s):=F_{m}(s), \\
P_{s}(s) X_{s}(s):=F_{s}(s) .
\end{gathered}
$$

The input of the master plant is the master force $F_{m}=$ $F_{h}-F_{m c}$, in the Laplace domain, where $F_{h}$ is the unknown force applied by the human operator and $F_{m c}$ the force from the controller. The plant has two outputs: $y_{m}$, the $n_{y}$ signals

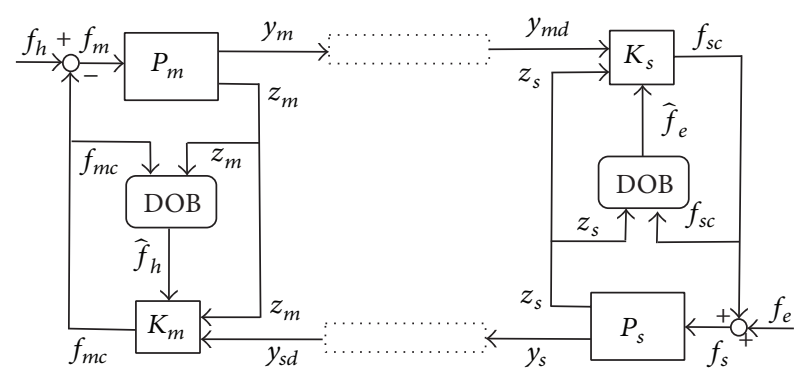

Figure 1: Generic teleoperation system.

that send information to the slave, and $z_{m}$, the $n_{z}$ signals used by the local controller.

The slave side can be treated in a similar way, where $F_{e}$ is the unknown force from the environment and the controller force $F_{s c}$ entering additively in $F_{s}=F_{e}+F_{s c}$.

In this work we also deal with the case of interaction with the environment on the slave side for implementation in applications such as robot arms with interacting tools. This can be described as

$$
\begin{aligned}
f_{e} & =-k_{e} x_{s}-B_{e} \dot{x}_{s}, \\
F_{e}(s) & =\left(-k_{e}-B_{e} s\right) X_{s}(s):=W(s) X_{s}(s) .
\end{aligned}
$$

In order to define how the signals sent from the master reach to the slave and vice versa, the delay parameter is assumed to be (see [8]) an unknown time-varying function for which the upper bounds on the magnitude and the variation satisfy $\forall t \geq 0$ :

$$
\begin{aligned}
0 & \leq \tau(t)=h+\eta(t) \leq h_{\max }, \quad|\eta(t)| \leq \kappa \leq h \\
|\dot{\tau}(t)| & \leq d<1 .
\end{aligned}
$$

In this way, we assume that the main effect of the communication channel is to introduce a time-varying delay in the form:

$$
\begin{gathered}
y_{m d_{i}}(t)=\mathscr{D}_{m}\left(y_{m_{i}}\right)(t):=y_{m i}\left(t-\tau_{m}(t)\right), \\
y_{s d_{i}}(t)=\mathscr{D}_{s}\left(y_{s_{i}}\right)(t):=y_{s i}\left(t-\tau_{s}(t)\right),
\end{gathered}
$$

where $\mathscr{D}_{m}$ and $\mathscr{D}_{s}$ are the corresponding time-delay operators.

Now, we consider the expressions for the Generalized four-channel control scheme (G-4C) in the most general and representative case of delay-dependent schemes. Here, the master and slave exchange, through the communication channel, the velocities, or positions and the forces in both directions, and the local controllers are defined in terms of the applied external force $\left(F_{h}, F_{e}\right)$, its own velocity/position $z_{m, s}$ (master/slave), and the delayed velocity/position and force $y_{s d, m d}$ (slave/master) from the other side.

In the classical notation $[1,2,4]$ the controllers $K_{m}$, $K_{s}$, when the system exchanges positions and forces (see Figure 2), are usually defined as

$$
\begin{gathered}
F_{m c}=C_{m} x_{m}+C_{2} F_{s d}+C_{4} x_{s d}-C_{6} F_{h}, \\
F_{s c}=-C_{s} x_{s}+C_{3} F_{m d}+C_{1} x_{m d}+C_{5} F_{e} .
\end{gathered}
$$




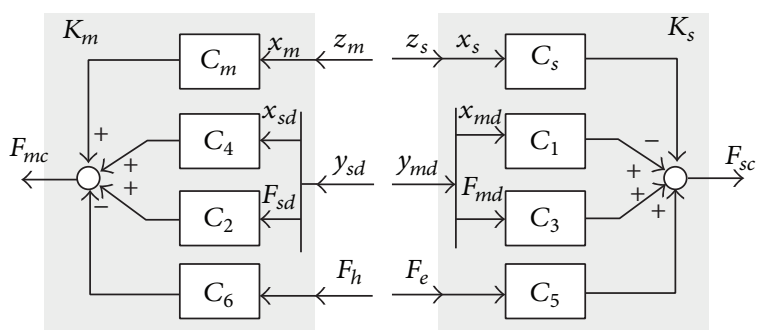

FIGURE 2: Local controllers in G-4C control scheme.

In this work we include the DOB blocks representing disturbance observers that estimate the external forces $F_{h}, F_{e}$ based on measurements of local controller forces $F_{m c}, F_{s c}$ and the mechanical systems coordinates (positions) $x_{m}, x_{s}$. Note that the use of DOBs in the control scheme involves obtaining the local controller forces $F_{m c}, F_{s c}$ in (5a) and (5b) from the estimated external forces $\widehat{F}_{h}, \widehat{F}_{e}$. This issue will be described in the following subsection.

2.1. Disturbance Observers DOB. A classical scheme for a disturbance observer [15] can be seen in Figure 3(a). The unknown signal $d$, an additive disturbance input to any plant $\mathscr{G}$, can be estimated from the known signals $u, x$ given an approximate model $\widehat{\mathscr{G}}$ of the plant by tuning the controller $K_{o}$.

Proposition 1. A DOB as defined in Figure 3(a), with the transfer function $\mathscr{G}$ as the plant and the transfer function $\widehat{\mathscr{G}}$ as the model, can be described by the equivalent diagram in Figure 3(b) with the following assignments:

$$
\begin{aligned}
& \Delta_{e}:=\mathscr{G}-\widehat{\mathscr{G}} \equiv \text { modeling error } \\
& \Delta_{1}:=\widehat{\mathscr{G}}^{-1} \mathscr{G}-I \equiv \text { multiplicative error. }
\end{aligned}
$$

Proof. The estimated signal $\widehat{d}$ in Figure $3(\mathrm{a})$ is

$$
\widehat{d}=-K_{o} \widehat{\mathscr{G}} \widehat{d}-K_{o} \widehat{\mathscr{G}} u+K_{o} \mathscr{G} d+K_{o} \mathscr{G} u
$$

Rearranging the terms and adding $\pm K_{o} \widehat{\mathscr{G}} d$,

$$
\left(I+K_{o} \widehat{\mathscr{G}}\right) \cdot \widehat{d}=-K_{o} \widehat{\mathscr{G}} u+K_{o} \mathscr{G} d+K_{o} \mathscr{G} u \pm K_{o} \widehat{\mathscr{G}} d,
$$

we get

$$
\left(I+K_{o} \widehat{\mathscr{G}}\right) \cdot \widehat{d}=K_{o} \widehat{\mathscr{G}} d+K_{o}(\mathscr{G}-\widehat{\mathscr{G}}) \cdot(u+d)
$$

and consequently,

$$
\Longrightarrow\left(I+K_{o} \widehat{\mathscr{G}}\right) \cdot \widehat{d}=K_{o} \widehat{\mathscr{G}} d+K_{o} \widehat{\mathscr{G}} \Delta_{1}(u+d) .
$$

Then, the following equation can be described in an equivalent block diagram as shown in Figure 3(b):

$$
\widehat{d}=\left(I+K_{o} \widehat{\mathscr{G}}\right)^{-1} K_{o} \widehat{\mathscr{G}} \cdot\left[\left(1+\Delta_{1}\right) \cdot d+\Delta_{1} \cdot u\right] .
$$

In this way, if the modelling error $\Delta_{e} \rightarrow 0$, that is $\widehat{\mathscr{G}} \rightarrow$ $\mathscr{G}, \Delta_{1} \rightarrow 0$ also, and it is possible to design $K_{o}$ so that $\widehat{d} \rightarrow d$ quickly and robustly.

Once Proposition 1 is proved, we apply this result to define the master and slave disturbance observers in the teleoperation system shown in Figure 1.

Definition 2. For $u=-F_{m c}, d=F_{h}$, the controller $K_{o}=K_{m_{o}}$, the master plant $\mathscr{G}=P_{m}^{-1}$, the master model $\widehat{\mathscr{G}}=\widehat{P}_{m}^{-1}$, and $\Delta_{1 m}:=\widehat{P}_{m}^{-1} P_{m}-I$, the external force $F_{h}$ can be estimated by a disturbance observer as in Figure 3 defined in the following form:

$$
\widehat{F}_{h}=\mathscr{F}_{m_{o}} \cdot\left[\left(I+\Delta_{1 m}\right) \cdot F_{h}-\Delta_{1 m} \cdot F_{m c}\right]
$$

with the closed loop transfer function:

$$
\mathscr{F}_{m_{o}}=\left(I+K_{m_{o}} \widehat{P}_{m}^{-1}\right)^{-1} K_{m_{o}} \widehat{P}_{m}^{-1} .
$$

Definition 3. For $u=F_{s c}, d=F_{e}$, the controller $K_{o}=K_{s_{o}}$, the slave plant $\mathscr{G}=P_{s}^{-1}$, the slave model $\widehat{\mathscr{G}}=\widehat{P}_{s}^{-1}$, and $\Delta_{1 s}:=\widehat{P}_{s}^{-1} P_{s}-I$, the external force $F_{e}$ at the slave side can be estimated by a disturbance observer as in Figure 3 defined in the following form:

$$
\widehat{F}_{e}=\mathscr{F}_{s_{o}} \cdot\left[\left(I+\Delta_{1 s}\right) \cdot F_{e}+\Delta_{1 s} \cdot F_{s c}\right]
$$

with the closed loop transfer function:

$$
\mathscr{F}_{s_{o}}=\left(I+K_{s_{o}} \widehat{P}_{s}^{-1}\right)^{-1} K_{s_{o}} \widehat{P}_{s}^{-1} .
$$

Remark 4. A first contribution of this work is that the DOB blocks required to observe the unknown external forces $F_{h}, F_{e}$ based on measurements of local controller forces $F_{m c}, F_{s c}$ and the positions $x_{m}, x_{s}$ can be described by the equivalent diagram in Figure 3(b) developed in Proposition 1, by representing the observers as stable, fast filters affected by the uncertainty in the plant modelling. Using Definition 2 and (12a) and (12b) we obtain the estimation of $F_{e}$ by DOB on the master side, and using Definition 3 and (13a) and (13b) we obtain the estimation of $F_{e}$ by the DOB on the slave side.

\section{Generic Model for Robust Stability in Delay-Dependent Control Schemes with Disturbance Observers}

Our first objective will be to remodel the whole system to obtain a single feedback loop containing a linear time invariant LTI block and an uncertain time-varying delay block. However, since teleoperation systems present several delayed signals and since time-varying delays do not commute with linear blocks, then it is not possible to reduce them to one single delay. As a consequence, the stability treatment requires the use of structured singular values $(\mu$ values) instead of ordinary singular values.

So, our strategy will be to rearrange the terms in such a way that the loop transformation of the delayed system maintains an uncertain time-varying delay block with the same 


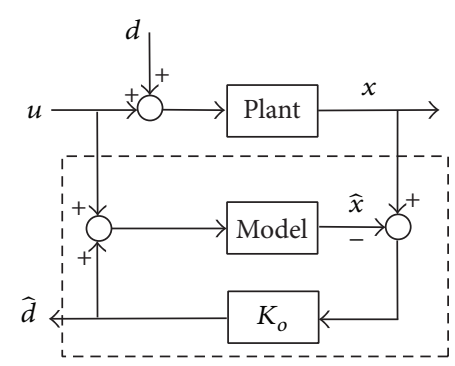

(a)

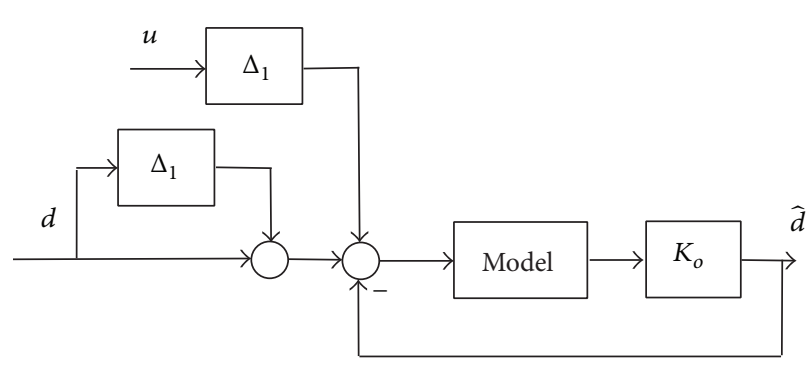

(b)

Figure 3: (a) Classical DOB scheme. (b) Equivalent diagram of DOB.

dimension as the number of the exchanged signals between the master and slave.

In previous works we have obtained these models for teleoperation systems with a 2C-position error control scheme [8], with a 4C-control scheme [14] and now, in this study, we model a teleoperation system with a general $4 \mathrm{C}$-control scheme incorporating the disturbance observers.

Proposition 5. A teleoperation setup as described in Figures 1, 2, and 3, given by (1)-(5b), (12a), (12b), (13a), and (13b), can be formulated as a compact model for robust stability analysis using the generic approach developed in [8] in the form given by (14), (15a), and (15b) and the block diagram in Figure 4:

$$
\Pi_{o}(s) X(s)=-C_{o}(s) \mathscr{D D}(s) X(s)+\Phi_{o}(s) F(s),
$$

with

$$
\begin{aligned}
& \Pi_{o}=\left(\begin{array}{cc}
P_{m}+\theta_{m} C_{m} & 0 \\
0 & \Pi_{22}
\end{array}\right) \\
& X=\left(\begin{array}{c}
X_{m} \\
X_{s}
\end{array}\right) \text {, } \\
& C_{o}=\left(\begin{array}{ccc}
0_{1 \times 2} & \theta_{m} C_{4} & \theta_{m} C_{2} \\
-\theta_{s} C_{1}-\theta_{s} C_{3} & 0_{1 \times 2}
\end{array}\right) \text {, } \\
& \mathscr{D}=\left(\begin{array}{cc}
I_{2} \cdot \mathscr{D}_{m} & 0_{2 \times 2} \\
0_{2 \times 2} & I_{2} \cdot \mathscr{D}_{s}
\end{array}\right) \text {, } \\
& D=\left(\begin{array}{cc}
\left(\begin{array}{cc}
1 & P_{m}
\end{array}\right)^{T} & 0_{2 \times 1} \\
0_{2 \times 1} & \left(\begin{array}{ll}
1 & P_{s}
\end{array}\right)^{T}
\end{array}\right), \\
& \Phi_{o}=\left(\begin{array}{cc}
1+\theta_{m} C_{6} \mathscr{F}_{m_{o}}\left(1+\Delta_{1 m}\right) & 0 \\
0 & \Phi_{22}
\end{array}\right) \text {, } \\
& F=\left(\begin{array}{c}
F_{h} \\
F_{e}
\end{array}\right)
\end{aligned}
$$

where $\theta_{m}:=\left(1-C_{6} \mathscr{F}_{m_{o}} \Delta_{1 m}\right)^{-1}, \theta_{s}:=\left(1-C_{5} \mathscr{F}_{s_{o}} \Delta_{1 s}\right)^{-1}$ and where the matrices $\Pi_{o}$, $\Phi_{o}$ take the following values depending on the case of external force as a disturbance $F_{e} \neq f\left(x_{s}\right)$

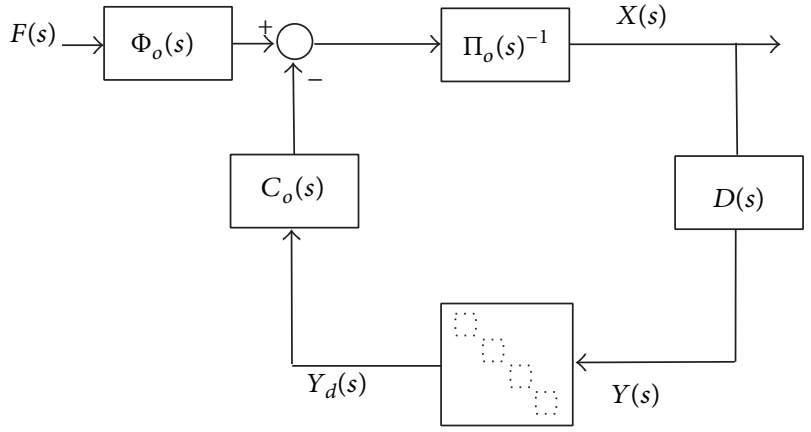

FIGURE 4: Teleoperation loop.

(including $F_{e}=0$ for free motion), or the case of environment as impedance Win (2):

Environment as disturbance case: $\Pi_{22}=P_{s}+\theta_{s} C_{s}$,

$\Phi_{22}=1+\theta_{s} C_{5}\left(1+\Delta_{1 s}\right) \mathscr{F}_{s_{o}}$

Environment as impedance case: $\Pi_{22}$

$$
=P_{s}+\theta_{s} C_{s}-\left[1+\theta_{s} C_{5}\left(1+\Delta_{1 s}\right) \mathscr{F}_{s_{o}}\right] W,
$$

$\Phi_{22}=0$.

Remark 6. Proposition 5 states one of the main results of this paper. A teleoperation setup with a general 4C-control scheme incorporating the disturbance observers can be formulated as a compact model for robust stability analysis by the loop transformation of the delayed system and maintains an uncertain time-varying delay block with the same dimension as the number of the exchanged signals between the master and slave.

We adopt the scaled four-channel control scheme $\gamma$-4C stated in our previous works $[6,14]$. The controllers in the $\gamma$-4C scheme incorporate a constant tuning factor $\gamma>1$, named the stability factor. The proposed form in which this factor is included in the controllers in (16) increases the stable region in practical conditions by fixing the desired bounds on time-varying delay, with the particularity of maintaining the tracking properties provided by this transparent control scheme. 
One of the contributions of this paper after considering these results is that we propose control based on observed external forces, to define the controllers in (5a) and (5b) in the form given by (16).

For $C_{m}(s), C_{s}(s), C_{5}, C_{6}$, and $\gamma>1$,

$$
\begin{aligned}
C_{4}(s) & =-\frac{\widehat{P}_{m}(s)+C_{m}(s)}{\gamma}, \\
C_{1}(s) & =\widehat{P}_{s}(s)+C_{s}(s), \\
C_{2} & =\frac{\left(1+C_{6}\right)}{\gamma}, \\
C_{3} & =\frac{\left(1+C_{5}\right)}{\gamma} .
\end{aligned}
$$

Definition 7. Given (14), (15a), and (15b) obtained by Proposition 5 and selecting a control scheme $\gamma$-4C with the values proposed in (16), we define the compact model for robust stability analysis in $\gamma-4 \mathrm{C}$ with disturbance-observerbased teleoperation as

$$
\Pi_{o}(s) X(s)=-C_{o}(s) \mathscr{D D}(s) X(s)+\Phi_{o}(s) F(s) .
$$

Obtained after suitable identification and given the controller values in (16). This model can be drawn by the blocks diagram in Figure 4.

Remark 8. The model described in Definition 7 is directly represented by Figure 4 and maintains four time-varying delays channels inherited from Figure 1, bearing in mind that they cannot be combined in a single delay because timevarying delays do not commute with LTI systems. The four delays are the ones affecting positions and forces sent from master to slave and back.

3.1. Steady-State Analysis. Note that from the teleoperation loop in Figure 4, we can also obtain the zero-delay $(\mathscr{D}=$ $I \Rightarrow Y_{d}=Y$ ) closed loop dynamics in order to study some transparency properties and the steady-state behavior, considering

$$
\begin{aligned}
& X(s) \\
& \quad=\left(I+\Pi_{o}^{-1}(s) C_{o}(s) D(s)\right)^{-1} \Pi_{o}^{-1}(s) \Phi_{o}(s) F(s) \\
& \quad=: \Lambda_{o}(s) F(s) .
\end{aligned}
$$

That is,

$$
\begin{aligned}
& x_{m}=\Lambda_{11} f_{h}+\Lambda_{12} f_{e}, \\
& x_{s}=\Lambda_{21} f_{h}+\Lambda_{22} f_{e},
\end{aligned}
$$

with the following values:

$$
\begin{aligned}
\Lambda_{11} & =\Delta_{\Lambda}{ }^{-1}\left(P_{m}+\theta_{m} C_{m}\right)^{-1} \\
& \left(1+\theta_{m} C_{6} \mathscr{F}_{m_{o}}\left(1+\Delta_{1 m}\right)\right), \\
\Lambda_{12} & =-\Delta_{\Lambda}{ }^{-1}\left(P_{m}+\theta_{m} C_{m}\right)^{-1} \theta_{m}\left(C_{4}+C_{2} P_{s}\right) \cdot \Pi_{22}^{-1} \\
& \cdot \Phi_{22}, \\
\Lambda_{21} & =\Delta_{\Lambda}{ }^{-1} \cdot \Pi_{22}^{-1} \cdot \theta_{s}\left(C_{1}+C_{3} P_{m}\right)\left(P_{m}+\theta_{m} C_{m}\right)^{-1} \\
& \cdot\left(1+\theta_{m} C_{6} \mathscr{F}_{m_{o}}\left(1+\Delta_{1 m}\right)\right), \\
\Lambda_{22} & =\Delta_{\Lambda}{ }^{-1} \cdot \Pi_{22}^{-1} \cdot \Phi_{22}
\end{aligned}
$$

with $\Delta_{\Lambda}=1+\Pi_{22}^{-1} \cdot \theta_{s}\left(C_{1}+C_{3} P_{m}\right)\left(P_{m}+\theta_{m} C_{m}\right)^{-1} \theta_{m}\left(C_{4}+C_{2} P_{s}\right)$ and $\Pi_{22}, \Phi_{22}$ as defined in (15b) for each $F_{e}$ case.

Regarding observers for analysis, if the modelling error $\Delta_{e} \rightarrow 0 \Rightarrow \Delta_{1 m}, \Delta_{1 s} \rightarrow 0$, and $K_{m_{o}}, K_{s_{o}}$ appropriately, we can obtain in the steady-state $\mathscr{F}_{m_{o}}, \mathscr{F}_{s_{o}} \rightarrow 1$. In these conditions, $\theta_{m}, \theta_{s} \rightarrow 1$.

Remark 9. Therefore, the $\Lambda$ steady-state values are given by (20) when the teleoperation setup is formed by master and slave systems with one degree-of-freedom, whose dynamic models are $P_{m}=M_{m} s^{2}+B_{m} s, P_{s}=M_{s} s^{2}+B_{s} s$ and tuning $C_{m}(s)=k_{m}, C_{s}(s)=k_{s}, C_{5}=$ constant, $C_{6}=$ constant and $\gamma$-4C controllers as in (16):

$$
\begin{array}{r}
\lim _{s \rightarrow 0} \Lambda(s)=\Lambda_{\infty} \\
=\frac{1}{\Delta_{\Lambda_{\infty}}}\left(\begin{array}{cc}
\frac{1+C_{6}}{C_{m_{\infty}}} & -\frac{C_{4_{\infty}}}{C_{m_{\infty}}} \frac{\Phi_{22_{\infty}}}{\Pi_{22_{\infty}}} \\
\frac{C_{1_{\infty}}}{\Pi_{22_{\infty}}} \frac{\left(1+C_{6}\right)}{C_{m_{\infty}}} & \frac{\Phi_{22_{\infty}}}{\Pi_{22_{\infty}}}
\end{array}\right), \\
\text { with } \Delta_{\Lambda_{\infty}}=1+\frac{C_{1_{\infty}} C_{4_{\infty}}}{C_{m_{\infty}} \Pi_{22_{\infty}}} .
\end{array}
$$

So that, depending on $F_{e}$, we obtain

Disturbance case: $\left.\Lambda_{\infty}\right|_{F_{e} \neq f\left(x_{s}\right)}$

$$
\begin{aligned}
& =\frac{1}{\Delta_{\Lambda_{\infty}}}\left(\begin{array}{cc}
\frac{1+C_{6}}{C_{m_{\infty}}} & -\frac{C_{4_{\infty}}}{C_{m_{\infty}}} \frac{\left(1+C_{5}\right)}{C_{s_{\infty}}} \\
\frac{C_{1_{\infty}}}{C_{m_{\infty}}} \frac{\left(1+C_{6}\right)}{C_{s_{\infty}}} & \frac{1+C_{5}}{C_{s_{\infty}}}
\end{array}\right) \\
& =\frac{\gamma}{\gamma-1}\left(\begin{array}{cc}
\frac{1+C_{6}}{k_{m}} & \frac{1}{\gamma} \frac{\left(1+C_{5}\right)}{k_{s}} \\
\frac{\left(1+C_{6}\right)}{k_{m}} & \frac{1+C_{5}}{k_{s}}
\end{array}\right),
\end{aligned}
$$




$$
\begin{aligned}
& \text { Impedance case: }\left.\Lambda_{\infty}\right|_{F_{e}=f\left(x_{s}\right)} \\
& =\frac{1}{\Delta_{\Lambda_{\infty}}}\left(\begin{array}{cc}
\frac{1+C_{6}}{C_{m_{\infty}}} & 0 \\
\frac{C_{1_{\infty}}}{C_{m_{\infty}}} \frac{\left(1+C_{6}\right)}{\left(C_{s_{\infty}}+k_{e}\left(1+C_{5}\right)\right)} & 0
\end{array}\right) \\
& =\frac{k_{e}\left(1+C_{5}\right)}{k_{s}+k_{e}\left(1+C_{5}\right)}\left(\begin{array}{cc}
\frac{1+C_{6}}{k_{m}} & 0 \\
\frac{k_{s}}{k_{m}} \frac{\left(1+C_{6}\right)}{\left(k_{s}+k_{e}\left(1+C_{5}\right)\right)} & 0
\end{array}\right) \text {, } \\
& \text { with } \Delta_{\Lambda_{\infty}}=1+\frac{C_{1_{\infty}} C_{4_{\infty}}}{C_{m_{\infty}}\left(C_{s_{\infty}}+k_{e}\left(1+C_{5}\right)\right)} \text {. }
\end{aligned}
$$

\section{Robust Stability for Delay-Dependent Control Schemes with Disturbance Observers}

In this Section, the approach to stability under time-varying delay in teleoperation developed in [8] is applied to the $\gamma-4 \mathrm{C}$ control scheme incorporating the external forces disturbance observers. A basic result adapted from [19] providing a bound of a certain delay subsystem, is combined with input-output stability criteria and $\mu$-analysis and synthesis techniques to reach a final robust stability condition (Theorem 11).

Proposition 10. A teleoperation setup as stated in Definition 7 and Figure 4 can be modeled as a negative single feedback loop containing a linear time invariant LTI block $G(s)$ and an uncertain time-varying delay block $\mathscr{D}$ shown in Figure 5(a), with

$$
G(s)=D(s) \Pi_{o}^{-1}(s) C_{o}(s)
$$

And so, the LTI subsystem considering (14), (15a), (15b), and (17) is

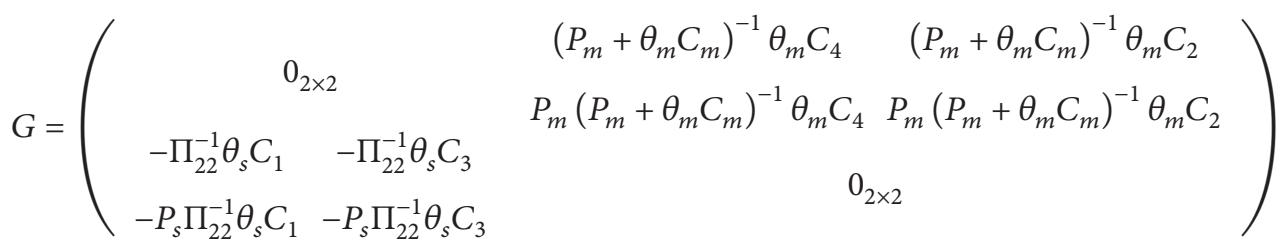

given the controller values in (16) and $\Pi_{22}, \Phi_{22}$ as defined in (15b) for each $F_{e}$ case.

Proof. Consider the feedforward action $\Phi_{o}(s)$ defined in (15a) and (15b). If the DOBs modelling error $\Delta_{e} \rightarrow 0 \Rightarrow$ $\Delta_{1 m} \Delta_{1 s} \rightarrow 0$ and it is possible to design $K_{o}$ so that estimated external forces tend to real ones quickly and robustly, then $\mathscr{F}_{m_{o}}, \mathscr{F}_{s_{o}} \rightarrow 1, \theta_{m}, \theta_{s} \rightarrow 1$. Also, it is natural to assume that $C_{5}$ and $\stackrel{C}{6}_{6}$ are designed such that $\Phi_{o}(s)$ is stable. Then the stability of the whole teleoperation system does not depend on $\Phi_{o}(s)$ and depends essentially on the stability of the feedback loop in Figure 4. Therefore, from (17) and Figure 4, $X=-\Pi_{o}^{-1} C_{o} Y_{d}$ and $Y=-D \Pi_{o}^{-1} C_{o} Y_{d}=-G Y_{d}$, which can be represented in a compact form in Figure 5(a).

Theorem 11. Consider a $\gamma-4 C$ based teleoperation system incorporating the external forces disturbance observers modeled by (14)-(17) and directly represented by Figure 4 and transformed into Figure 5(a) by Proposition 10. Let $G(s)$ be as defined in (23) and let $\phi(s)$ be as defined in [19]

$$
\phi(s)=k \cdot \frac{h_{\max }^{2} s^{2}+c \cdot h_{\max } s}{h_{\max }^{2} s^{2}+a h_{\max } s+k \cdot c}
$$

with $k=1+1 / \sqrt{1-d}, a=\sqrt{2 k \cdot c}$, where $c$ is any positive real number and delay bounds $h_{\max }$, $d$ are as defined in (3a) and (3b).
A sufficient condition for stability of the delayed system as described in Figure 5(a), with a complex diagonal structured uncertainty, is

$$
\Gamma=\max _{\omega \in[0, \infty]} \bar{\mu}[H(j \omega)]=\max _{\omega \in[0, \infty]} \rho[H(j \omega)]<1,
$$

where

$$
H(s)=: \phi(s) \cdot(I+G(s))^{-1} G(s)
$$

and $\bar{\mu}(\cdot)$ is the structured singular value (SSV) with respect to a repeated complex scalar uncertainty, which is equal to $\rho(\cdot)$, the spectral radius of a matrix (the maximum of the norms of the eigenvalues).

Proof. This result is obtained first, by applying the loop transformation theorem (Figure 5(a) loop is stable iff Figure 5(b) loop is stable) and the small gain theorem to Figure 5(b), whereby $\|H(s)\|_{L_{2}} \cdot\|\Delta\|_{L_{2}}<1$. Since $\|\Delta\|_{L_{2}} \leq 1$ by [19], the sufficient condition is $\|H(s)\|_{L_{2}}<1$. Second, using the $\mu$-techniques for robust stability [20] we can interpret Figure 5(b) as a nominal system $H(s)$ connected to a dynamic or complex uncertainty $\Delta$ that is actually diagonal. More details can be seen in [8].

Remark 12. As a prerequisite for robust stability, we must first satisfy nominal stability; that is, $H(s)$ must be internally stable. A necessary condition for this, derived from $H(s)$ definition (26), is $|I+G(s)| \neq 0$. 


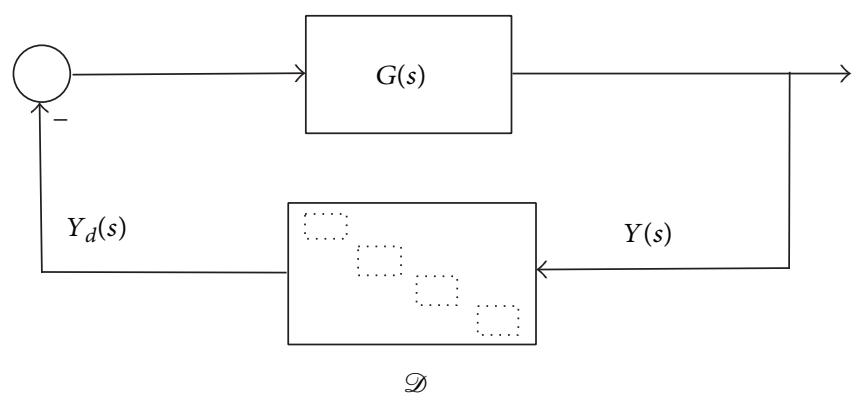

(a)

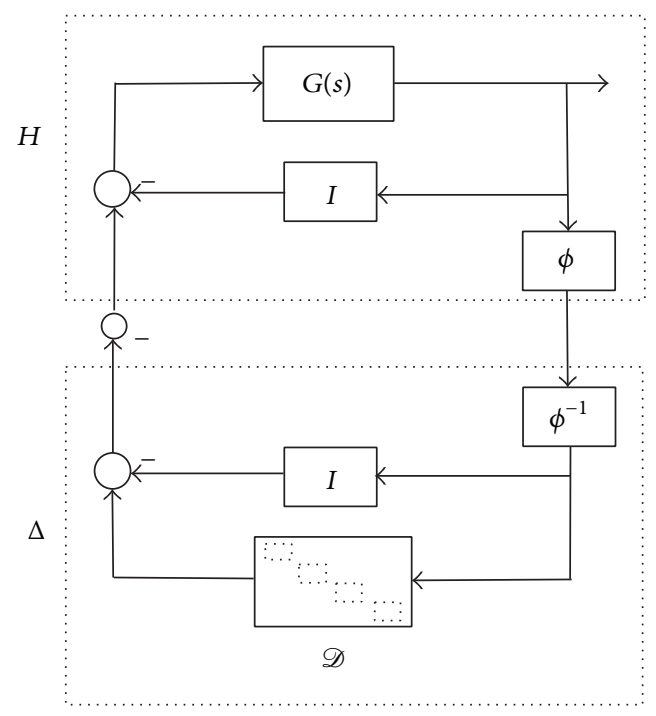

(b)

FIGURE 5: (a) LTI system with time-varying delay in the feedback loop. (b) Loop transformation of the delay system.

The use of frequency techniques combined with inputoutput stability criteria and $\mu$-analysis and synthesis techniques provides properties of robustness. Therefore using the stability condition (25) for any delay satisfying (3a) and (3b), we ensure robust stability under time-varying delays. But the modeled systems can also present a second uncertainty: the variation of the parameters, and this has an influence on system $H(s)$ in the final condition (25). Parameter variation could be treated explicitly in the $\mu$-synthesis framework although it can be simply addressed by discretization of the parameter intervals and by checking (25) for the mesh of discretized values. This is less conservative, as recommended in [20], and also provides robustness against parametric uncertainty.

In our teleoperation case study with the control scheme incorporating the external forces disturbance observers, the parametric uncertainties are dealt with by using different models for the nominal plants and for the plant estimations used in the observers and controllers.

Remark 13. As the main contribution of this paper, Proposition 10 remodels the $4 \mathrm{C}$ control scheme to incorporate the external forces disturbance observers to obtain a single feedback loop containing a linear time invariant LTI block and an uncertain time-varying delay block to reach a final robust stability condition, proposed in Theorem 11 through (25), over the whole system.

\section{Teleoperation Case Study}

The previous generalized framework for $4 \mathrm{C}$ architecture with force observers through DOBs has been analyzed by simulations using Simulink and Matlab. In this section we describe the parameters of the simulation case study. The following section presents the simulation results.
We study the remote teleoperation between master and slave haptic devices. The master and slave sides consist of identified linear models of two Phantom Omni haptic devices manufactured by SensAble Technologies Inc. These devices have 6-DoF position sensing and 3-DoF force actuation. To focus on the robust stability issue, rather than multiDoF issues, we limit the movements to 1-DoF. This has been achieved using the first rotational coordinate (the base rotation) as the free DoF. In this way, the two Omnis rotate around their vertical axes, while the shoulder, elbow, and stylus are blocked.

Thus, the master and slave positions are $\left(x_{m}, x_{s}\right)$, the sensed Omni base angles in radians (rad). The actuations are $\left(f_{m}, f_{s}\right)$, the torque inputs to the motors for vertical axis rotation, in machine units $(\mathrm{mu})$ because we do not have access to physical units.

We concentrate on 1-DoF movements around a fixed position (the Omnis' "zero"). Although the Omnis are robotic nonlinear systems, it is expected from the linearization principle that the local dynamics from forces to positions can be captured by models in the form $G_{i}=P_{i}^{-1}=1 / s\left(M_{i} s+B_{i}\right)$, as usual [21]. Notice that here we maintain the terms "forces" and "positions" for simplicity, to denote also torques and angles. Notice as well that since the forces are in machine units, the inertia-mass $M$ and friction $B$ coefficients will be given in machine or virtual units.

After linearizing the devices by dead-zone compensation, the linear models $G_{i}(s)=1 /(s(M s+B))$ can be obtained as usual [21] by applying in closed loop (proportional controller) square and triangular references, recording force inputs and position outputs and performing standard least-squares identifications of $M, B$. In this way, the simulated master and slave plants are $P_{m}^{-1}=1 /\left(M_{m} s^{2}+B_{m} s\right), P_{s}^{-1}=1 /\left(M_{s} s^{2}+\right.$ $B_{s} s$ ) (in machine units) with $M_{m}=M_{s}=0.0015$ (m.u.), $B_{m}=B_{s}=0.028$ (m.u.). For the observers in (12a)-(13b) and the controllers $\left(C_{1}, C_{4}\right.$ in (16)) we establish master and 
slave approximate models with the values $\widehat{P}_{m}^{-1}=\widehat{P}_{s}^{-1}=$ $1 /\left(0.001 \mathrm{~s}^{2}+0.02 \mathrm{~s}\right)$. We complete the controller parameters in (16) with the numerical values: $C_{m}=k_{m}, C_{s}=k_{s}$ with $k_{m}=k_{s}$ $=0.1 ; C_{5}=C_{6}=0.2, \gamma=10$.

Related to the external forces, the human forces $f_{h}$ will be ramp inputs simulating how the human operator moves the master in its free work space to be followed by the slave. But on the slave side, there is a "wall," hard $\left(k_{e}=4\right)$ or soft $\left(k_{e}=\right.$ $0.2)$, with damping $\left(B_{e}=0.2\right)$ or not, located at $x_{s}=0.3$ so that the slave will be in contact with the environment once it gets to that position, reflecting the perceived force to the master side.

These forces are estimated by DOBs in which the designed observer controllers are PID-type (proportional, integral, and derivative) controllers in the form

$$
K_{m_{o}}=K_{s_{o}}=\frac{6.25 \cdot(s+20)^{2}}{s \cdot(s+100)}
$$

to obtain $\widehat{d} \rightarrow d$ (in Figure 3(b), i.e., $\widehat{F}_{h} \rightarrow F_{h}$ and $\widehat{F}_{e} \rightarrow F_{e}$ ) with null steady-state error for step and ramp inputs in $d$ and with a fast transient (at least three times faster than the control transient), bandwidth $100 \mathrm{rad} / \mathrm{sec}$ and phase margin $41^{\circ}$.

Remark 14. If the DOBs modelling error tends to zero, $\Delta_{e} \rightarrow$ 0 , and the observer controllers are selected as in (27), fulfilling these design requirements, we ensure that estimated external forces tend to real ones with null error, quickly and robustly, and so $\mathscr{F}_{m_{o}}, \mathscr{F}_{s_{o}} \rightarrow 1, \theta_{m}, \theta_{s} \rightarrow 1$.

\section{Analysis and Simulation Results}

The robust stability tool under time-varying delay, described in Section 4, provides a sufficient condition for stability $\Gamma<$ 1 that depends on the delay bounds $\left(h_{\max }, d\right)$, on some parametric uncertainty $\left(\Delta_{e}\right)$, on the designed observers and on the $\gamma$ factor in local controllers in (16). The stability analysis can be assessed by applying Theorem 11 using Matlab. In this way, we compute the structured singular value (SSV) $\bar{\mu}[H(j \omega)]$ for $\omega \in(0, \infty)$ and test the stability by applying condition $\Gamma=\max _{\omega \in[0, \infty]} \bar{\mu}[H(j \omega)]<1$.

The influence of the $\gamma$ factor on the stability has been studied in previous works $[6,14]$. As the main conclusions, we highlighted that the stability factor lets us increase the stability margin in practical conditions, maintaining the tracking properties of the system, and we can adjust the $\gamma$ factor in order to ensure the stability of the system given the desired delay characteristics.

Here, for brevity, we present in Figure 6 the stability condition results for the teleoperation case study described in Section 5, with the DOBs in free motion $\left(F_{e}=0\right)$. First, fixing the delay bounds $h_{\max }=0.5 \mathrm{~s}$ and $d=0.45$ we show the SSV (red...) for $\gamma=3,5,7,9,10$, checking that the system is stable for $\gamma=10$ (red) because $\Gamma=0.9349<1$. Then we change the delay conditions with the same $\gamma=10$ : in green $(--) h_{\max }=$ $5 \mathrm{~s}, d=0.45 \Rightarrow \Gamma=1.0793$ and in blue $(-\cdot) h_{\max }=0.5 \mathrm{~s}, d=0.95$ $\Rightarrow \Gamma=1.9636$. The system is unstable in both cases.

Following this procedure, in Table 1 we present the minimum value of the stability factor $\gamma$ to ensure the stability

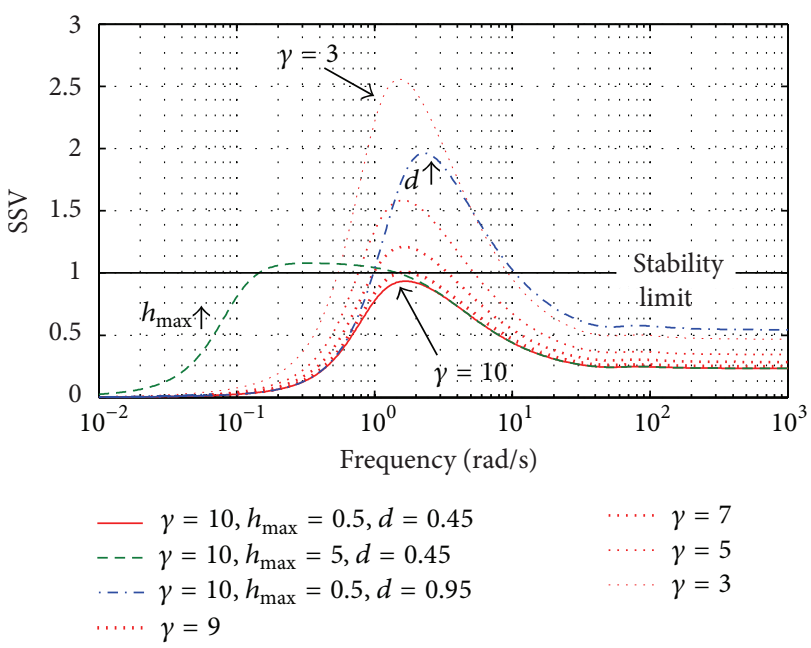

FIGURE 6: $\Gamma$ : Upper bound on $\mu$ for $d=0.45, h_{\max }=0.5, \gamma=3,5,7,9$, 10 (red), for $d=0.45, h_{\max }=5, \gamma=10$ (green - -), for $d=0.95, h_{\max }=$ $0.5, \gamma=10$ (blue --).

TABLE 1: Minimum stability factor for stability.

$\begin{aligned} & \text { Environment } \\ & \text { conditions }\end{aligned} f_{e}=-4 x_{s} \quad f_{e}=-4 x_{s}-0.2 \dot{x}_{s} \quad f_{e}=-0.2 x_{s} \quad f_{e}=0$

\begin{tabular}{lllll}
\hline Minimum $\gamma$ & 1 & 1.1 & 7.8 & 9.1 \\
\hline
\end{tabular}

of the teleoperation system with $h_{\max }=0.5 \mathrm{~s}$ and $d=0.45$ for different environment forces $F_{e}$.

Then, we study the performance of the system through simulations when the human operator applies a reference force $\left(F_{h}\right)$ to move the master and it is followed by the slave, which has in its work space $\left(x_{s}=0.3\right)$ a wall. For these simulation conditions, the observer on the slave side must only be applied when $F_{e} \neq 0$. This last issue can be understood by bearing the observer structure in Figure 3(b) in mind. When $\Delta_{e} \neq 0 \Rightarrow \Delta_{1} \neq 0$. On the slave side, the unknown signal $\left(d=F_{e}\right)$, when the slave is in free motion, is zero, and so the observer output $\widehat{d}$ will tend to known signal $u=F_{s c}$, with values to follow the master positions as the control system requires. Note that if this occurs on the master side, $d=0=$ $F_{h}$ implies that the control system reference is zero. Therefore the observer output will tend to known signal $u=-F_{m c}$ but in this case it will also be zero.

The simulation results are with delay values $h=0.5 \mathrm{~s}$, $|\eta(t)| \leq 0.01 \mathrm{~s}$, and $d=0.45$. Due to time-varying delay we saw in simulations that the master and slave forces were very noisy, which is not very desirable. To solve this issue, the output signals in $C_{1}, C_{4}$ controllers in (16) must be filtered. So, we select a second order lowpass butterworth filter with a cutoff frequency of $100 \mathrm{rad} / \mathrm{sec}$ at the output of $C_{1}, C_{4}$. The master/slave plants are not the same as the master/slave models; that is, $\Delta_{e} \neq 0$ with the values described in Section 5.

In Figure 7 the environment impedance is $F_{e}=-0.2 x_{s}$, and in Figure 8 we consider hard wall impedances. In this last case the contact generates high force oscillation that the observer is able to detect, which also tests the performance 


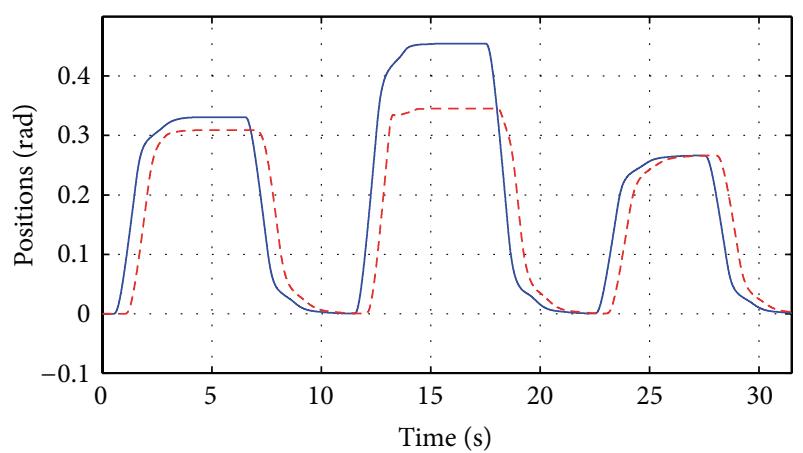

- Master

- - - Slave

(a) Master position (blue -) and slave position (red - -)

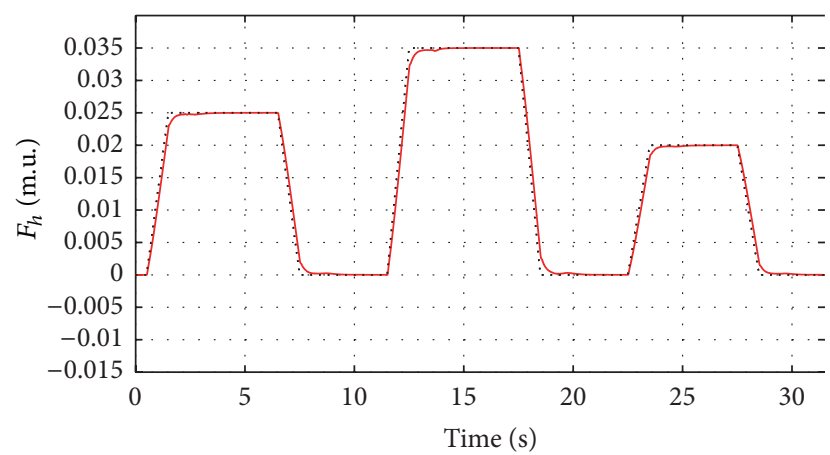

$\cdots \cdot F_{h}$

- Observed $F_{h}$

(c) Force reference $F_{h}$ (black $\cdot$ ), observed $\widehat{F}_{h} \rightarrow F_{h}$ (red -)

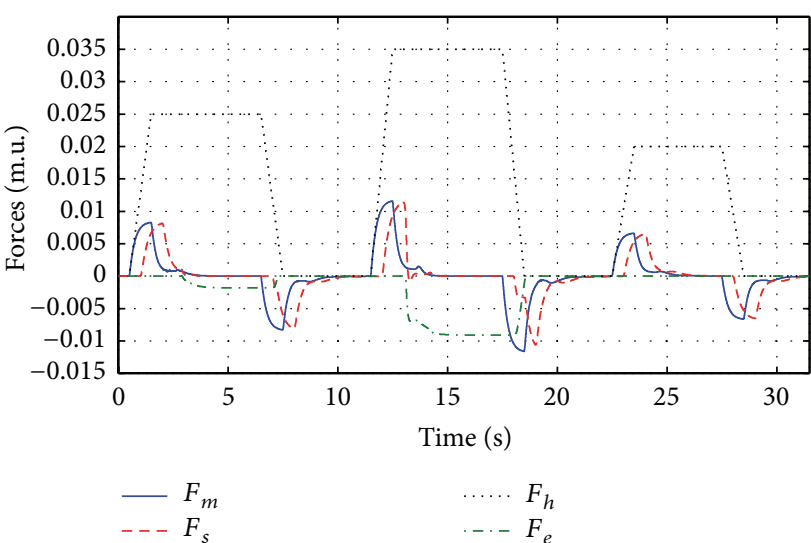

(b) Force reference $F_{h}$ (black $\left.\cdot \cdot\right), F_{e}$ (green -), master force (blue -), and slave force (red - -)

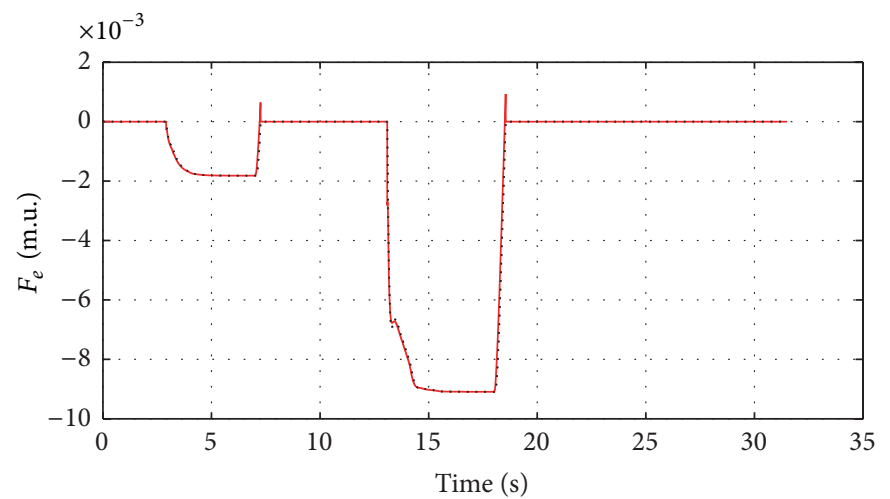

$F_{e}$

- Observed $F_{e}$

(d) Environment force $F_{e}$ (black $\cdot$ ), $\widehat{F}_{e} \rightarrow F_{e}$ observed (red -)

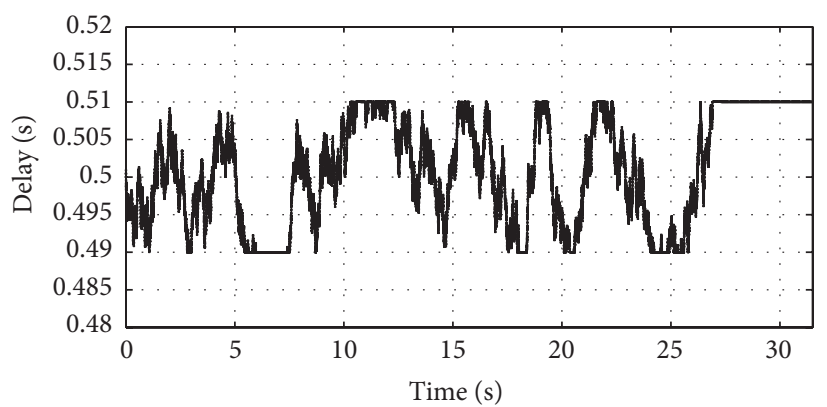

(e) Delay

FIGURE 7: Simulation case soft wall impedance: $\gamma=10$ with $h=0.5,|\eta(t)| \leq 0.01 \mathrm{~s}, d=0.45, \Delta_{e} \neq 0$.

of the system. We can conclude that the designed DOBs in which $\Delta_{e} \rightarrow 0$ are robust.

The interested reader can see in [22] more detailed simulations and an appendix with the proof of Proposition 5.

\section{Conclusions}

In this paper we have extended our previous framework for robust stability to the practical case in which there are no force measurements and thus DOBs are required to observe unknown forces and to implement the four-channel architecture. This has been achieved by representing the observers as stable, fast filters affected by the uncertainty in the plant modelling. In this way the stability test has an SSV formulation including the observer parameters. We have done extensive simulations with modelling errors, various delay ranges, jitter, and environments on the slave side, confirming the usefulness of the approach.

There are several possible extensions to this work to be considered for future research. To improve the teleoperation 


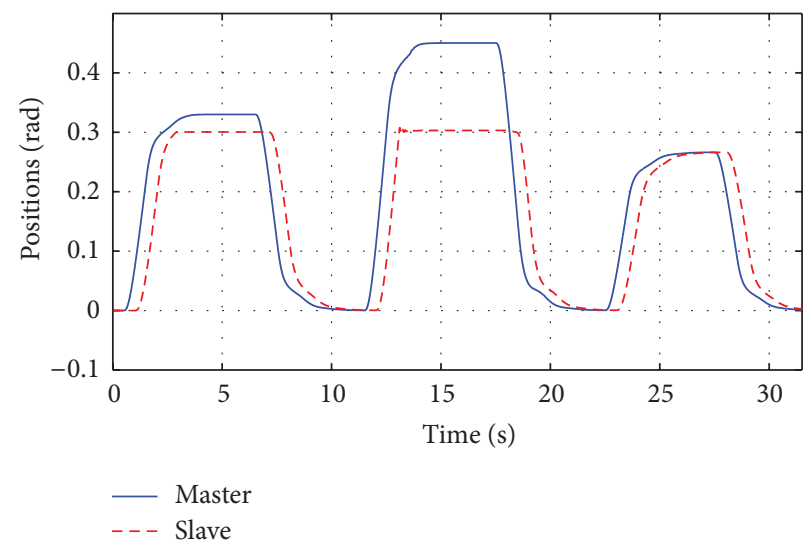

(a) Master position (blue -) and slave position (red - -)

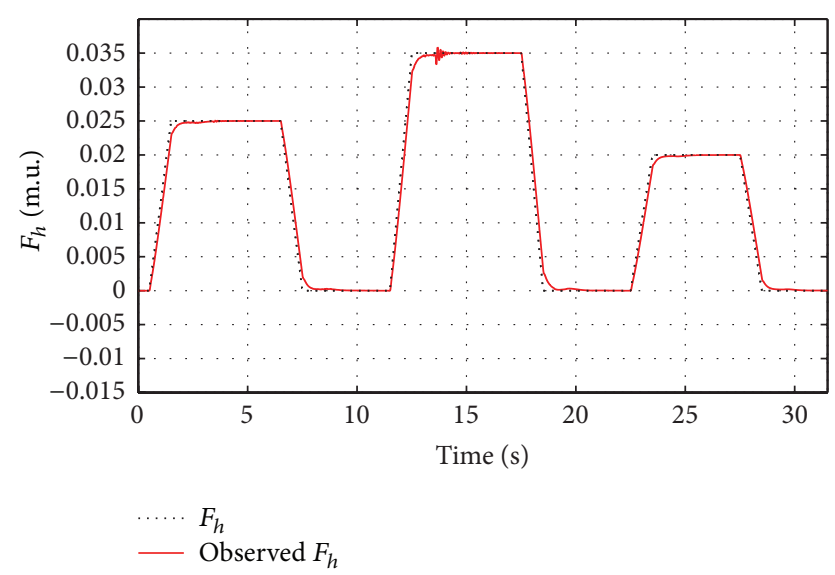

(c) Force reference $F_{h}$ (black $\cdot$ ), observed $\widehat{F}_{h} \rightarrow F_{h}$ (red -)

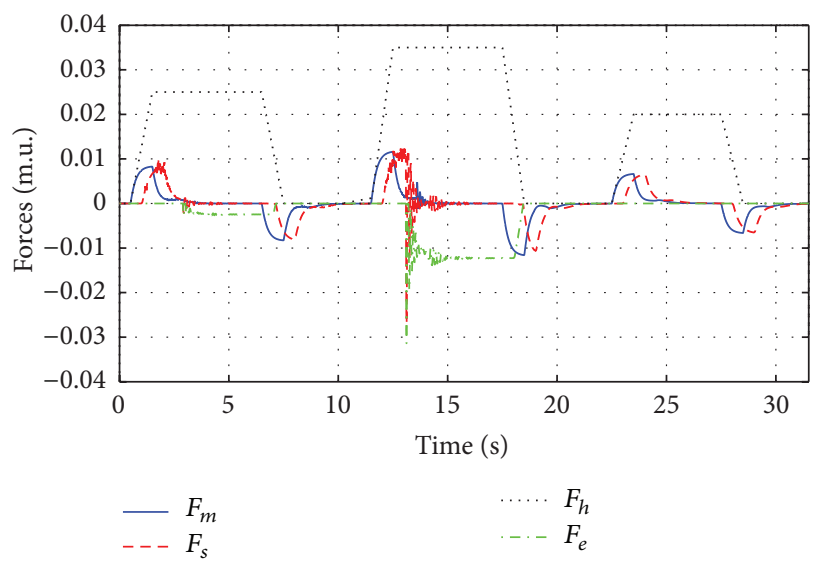

(b) Force reference $F_{h}$ (black *), $F_{e}$ (green -.), master force (blue -), and slave force (red - -)

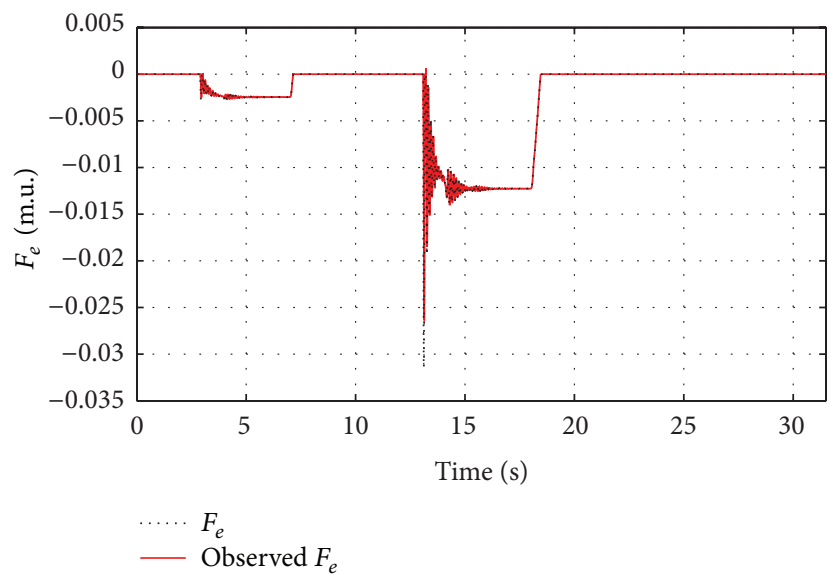

(d) Environment force $F_{e}$ (black $\cdot$ ), $\widehat{F}_{e} \rightarrow F_{e}$ observed (red -)

FIGURE 8: Simulation case hard wall impedance: $\gamma=10$ with $h_{\max }=0.5,|\eta(t)| \leq 0.01 \mathrm{~s}, d=0.45$.

robustness, for example, Lyapunov-Krasovskii functionals can be used as in [23] or the generic IQC framework as in $[24,25]$.

\section{Conflict of Interests}

The authors declare that there is no conflict of interests regarding the publication of this paper.

\section{Acknowledgment}

This work has been funded by MINECO (Spain) under the research Project DPI2013-47100-C2-2-P.

\section{References}

[1] D. A. Lawrence, "Stability and transparency in bilateral teleoperation," IEEE Transactions on Robotics and Automation, vol. 9, no. 5, pp. 624-637, 1993.

[2] P. Arcara and C. Melchiorri, "Control schemes for teleoperation with time delay: a comparative study," Robotics and Autonomous Systems, vol. 38, no. 1, pp. 49-64, 2002.
[3] R. J. Anderson and M. W. Spong, "Bilateral control of teleoperators with time delay," IEEE Transactions on Automatic Control, vol. 34, no. 5, pp. 494-501, 1989.

[4] K. Hashtrudi-Zaad and S. E. Salcudean, "Transparency in timedelayed systems and the effect of local force feedback for transparent teleoperation," IEEE Transactions on Robotics and Automation, vol. 18, no. 1, pp. 108-114, 2002.

[5] G. Niemeyer and J.-J. E. Slotine, "Stable adaptive teleoperation," IEEE Journal of Oceanic Engineering, vol. 16, no. 1, pp. 152-162, 1991.

[6] E. Delgado, M. Díaz-Cacho, and A. Barreiro, "Stability of teleoperation systems for time-varying delays by neutral LMI techniques," Mathematical Problems in Engineering, vol. 2012, Article ID 467629, 17 pages, 2012.

[7] K. Natori, R. Oboe, and K. Ohnishi, "Stability analysis and practical design procedure of time delayed control systems with communication disturbance observer," IEEE Transactions on Industrial Informatics, vol. 4, no. 3, pp. 185-197, 2008.

[8] E. Delgado, M. Díaz-Cacho, D. Bustelo, and A. Barreiro, "Generic approach to stability under time-varying delay in teleoperation: application to the position-error control of a gantry crane," IEEE/ASME Transactions on Mechatronics, vol.18, no. 5, pp. 1581-1591, 2013. 
[9] P. F. Hokayem and M. W. Spong, "Bilateral teleoperation: an historical survey," Automatica, vol. 42, no. 12, pp. 2035-2057, 2006.

[10] N. Chopra, M. W. Spong, and R. Lozano, "Synchronization of bilateral teleoperators with time delay," Automatica, vol. 44, no. 8, pp. 2142-2148, 2008.

[11] E. Nuño, L. Basañez, and R. Ortega, "Passivity-based control for bilateral teleoperation: a tutorial," Automatica, vol. 47, no. 3, pp. 485-495, 2011.

[12] Y. Nakajima, T. Nozaki, and K. Ohnishi, "Heartbeat synchronization with haptic feedback for telesurgical robot," IEEE Transactions on Industrial Electronics, vol. 61, no. 7, pp. 37533764, 2014.

[13] S. Dashkovskiy, M. Kosmykov, and F. R. Wirth, "A small-gain condition for interconnections of ISS systems with mixed ISS characterizations," IEEE Transactions on Automatic Control, vol. 56, no. 6, pp. 1247-1258, 2011.

[14] E. Delgado, M. Díaz-Cacho, P. Falcón, and A. Barreiro, "Robust stability in $\gamma$-4C based teleoperation," in Proceedings of the 39th Annual Conference of the IEEE Industrial Electronics Society (IECON '13), pp. 4258-4263, Vienna, Austria, 2013.

[15] K. Ohnishi, M. Shibata, and T. Murakami, "Motion control for advanced mechatronics," IEEE/ASME Transactions on Mechatronics, vol. 1, no. 1, pp. 56-67, 1996.

[16] E. Sariyildiz and K. Ohnishi, "An adaptive reaction force observer design," IEEE/ASME Transactions on Mechatronics, vol. 20, no. 2, pp. 750-760, 2015.

[17] C. Xiang, X. Wang, Y. Ma, and B. Xu, "Practical modeling and comprehensive system identification of a BLDC motor," Mathematical Problems in Engineering, vol. 2015, Article ID 879581, 11 pages, 2015.

[18] T. Murakami, F. Yu, and K. Ohnishi, “Torque sensorless control in multidegree-of-freedom manipulator," IEEE Transactions on Industrial Electronics, vol. 40, no. 2, pp. 259-265, 1993.

[19] C.-Y. Kao and A. Rantzer, "Stability analysis of systems with uncertain time-varying delays," Automatica, vol. 43, no. 6, pp. 959-970, 2007.

[20] S. Skogestad and I. Postlethwaite, Multivariable Feedback Control: Analysis \& Design, John Wiley \& Sons, New York, NY, USA, 1996.

[21] J. Huang, Y. Shi, and J. Wu, "Transparent virtual coupler design for networked haptic systems with a mixed virtual wall," IEEE/ASME Transactions on Mechatronics, vol. 17, no. 3, pp. 480-487, 2012.

[22] E. Delgado, P. Falcón, M. Díaz-Cacho, and A. Barreiro, "Internal report about Four-Channel Teleoperation with time-varying delays and Disturbance Observers".

[23] S. Islam, P. X. Liu, A. El Saddik, and Y. B. Yang, "Bilateral control of teleoperation systems with time delay," IEEE/ASME Transactions on Mechatronics, vol. 20, no. 1, pp. 1-12, 2015.

[24] I. Polat and C. W. Scherer, "Stability analysis for bilateral teleoperation: an IQC formulation," IEEE Transactions on Robotics, vol. 28, no. 6, pp. 1294-1308, 2012.

[25] H. Tugal, J. Carrasco, and M. Maya-Gonzalez, "Teleoperation with memoryless, monotone and bounded environments: a Zames-Falb multiplier approach," in Proceedings of the European Control Conference (ECC '15), Linz, Austria, 2015. 


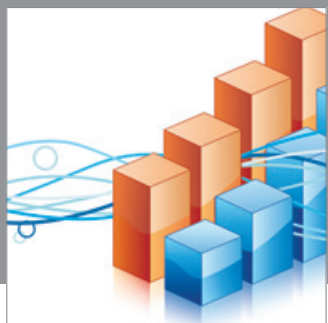

Advances in

Operations Research

mansans

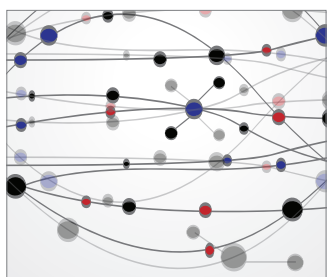

The Scientific World Journal
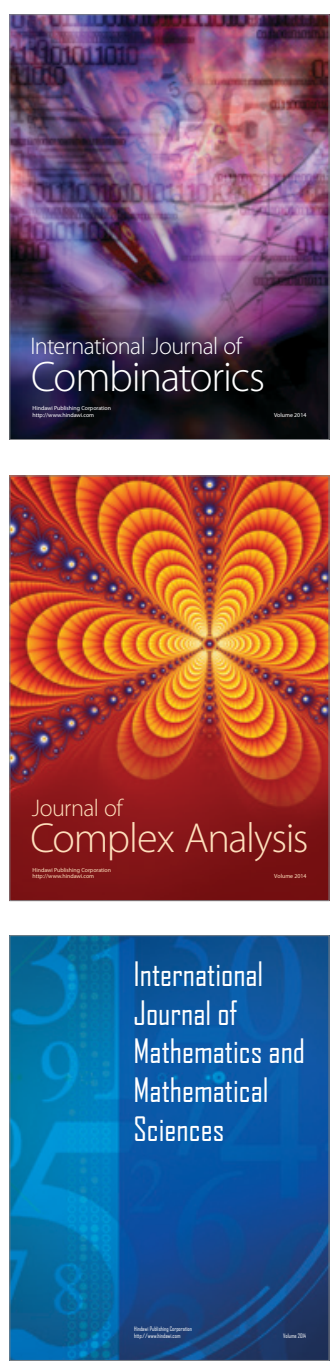
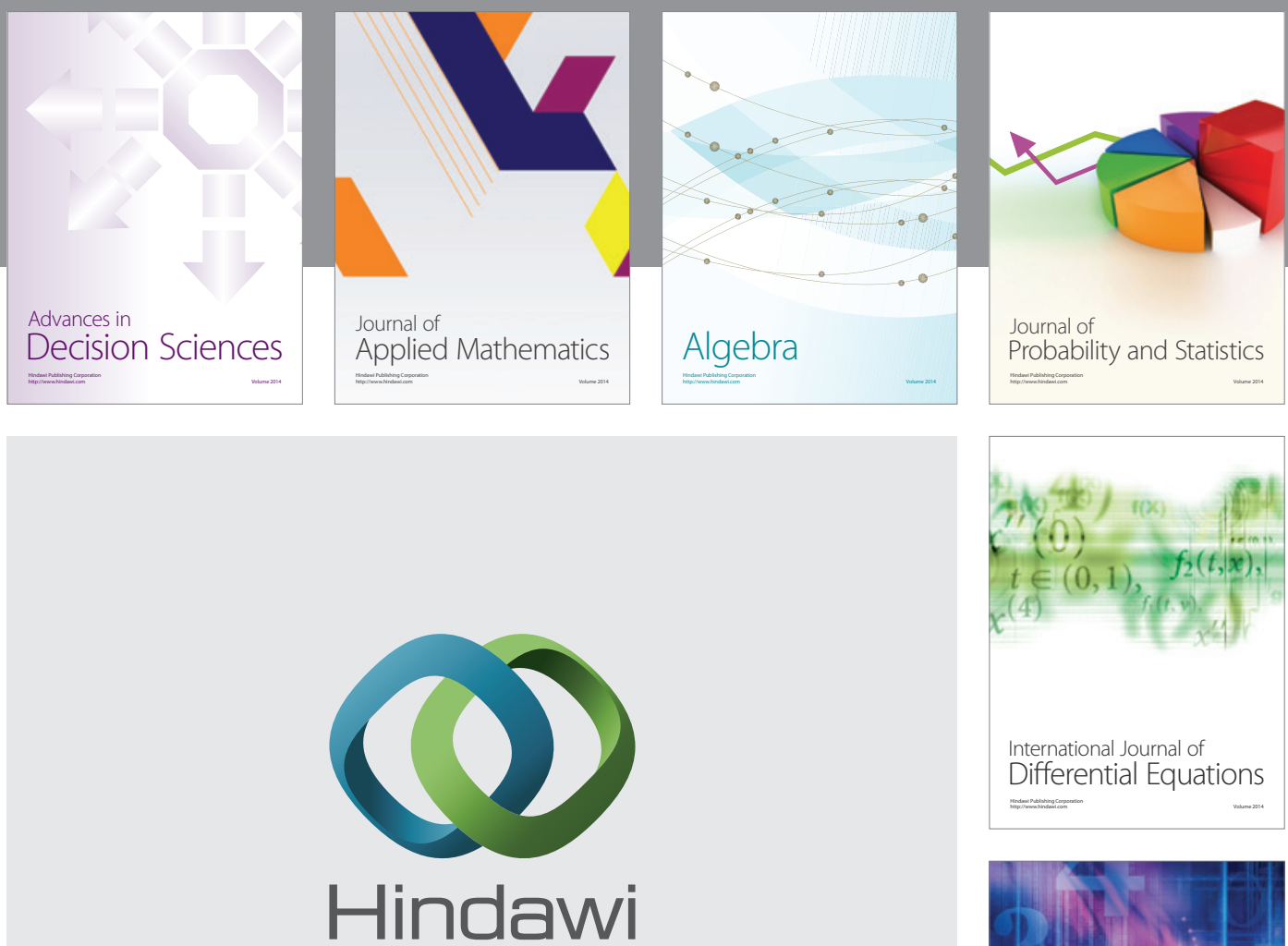

Submit your manuscripts at http://www.hindawi.com
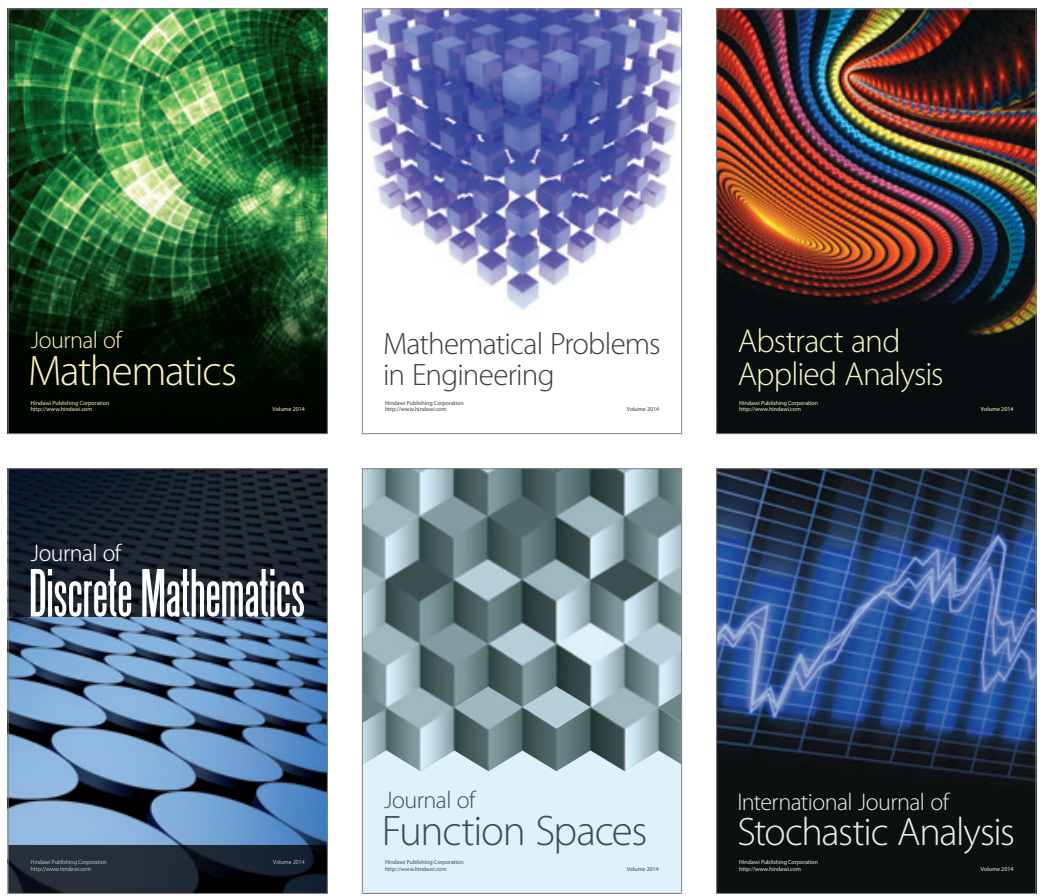

Journal of

Function Spaces

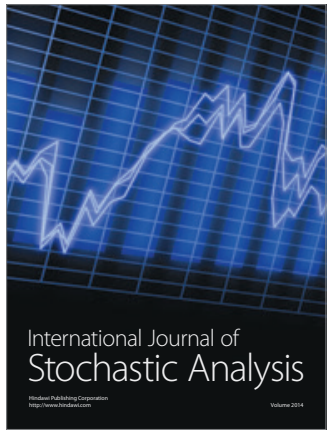

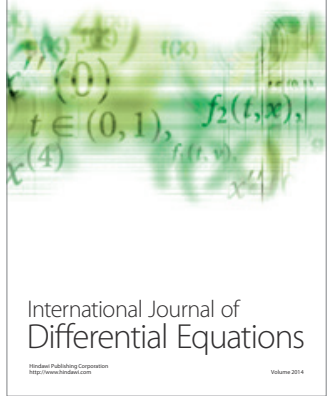
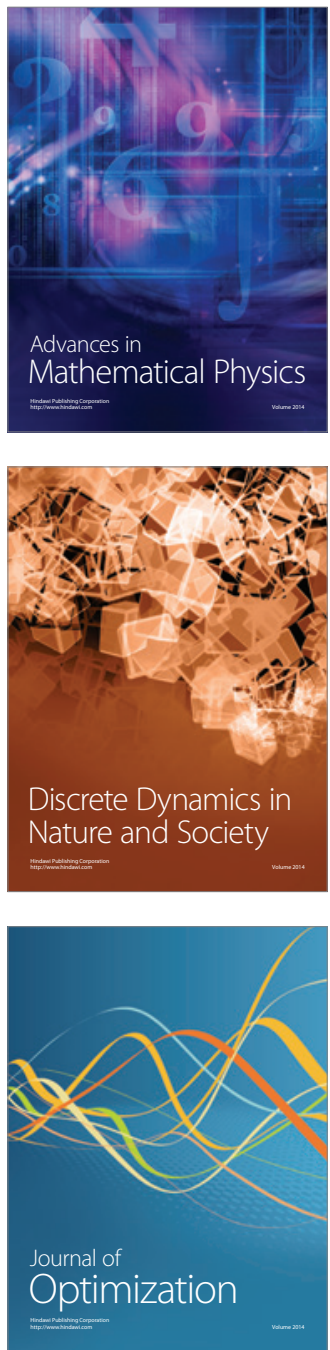